\title{
Des connaissances scientifiques en quête de connaissances d'acteurs
}

\author{
Elsa Faugère ${ }^{1}$, Mireille Navarrete ${ }^{2}$, Marie Charles ${ }^{1}$, Michel Étienne ${ }^{3}$, Joël Fauriel ${ }^{2}$, \\ Jacques Lasseur ${ }^{4}$, Élisabeth Lécrivain ${ }^{5}$, Martine Napoléone ${ }^{4}$, Réjane Paratte ${ }^{1}$ \\ 1 Ethnologues, INRA, Unité d'écodéveloppement, 84914 Avignon cedex 09, France \\ 2 Agronomes, INRA, Unité d'écodéveloppement, 84914 Avignon cedex 09, France \\ 3 Écologue, INRA, Unité d'écodéveloppement, 84914 Avignon cedex 09, France \\ 4 Zootechniciens, INRA, Unité d'écodéveloppement, 84914 Avignon cedex 09, France \\ 5 Écoéthologue, INRA, Unité d'écodéveloppement, 84914 Avignon cedex 09, France
}

Les connaissances que les acteurs mobilisent dans des pratiques routinières peuvent-elles servir à élaborer des connaissances
scientifiques? Voilà une question dont on s'attendrait qu'elle soit traitée par des spécialistes des sciences cognitives. Mais ce n'est
pas le cas ici. L'intérêt de l'article qui suit vient, nous semble-t-il, du travail réflexif d'un collectif de scientifiques de disciplines
différentes sur leur rapport aux connaissances d'acteurs. Qu'ils relèvent des sciences sociales, de celles de la nature ou de
l'action, les auteurs de ce texte ont en commun de travailler avec des acteurs pour faire émerger, par enquête ou observation, les
connaissances issues de la pratique. Qu'en ont-ils fait? C'est à cette question qu'ils ont réfléchi, en confrontant leurs expériences.

La Rédaction

\section{Mots-clés :}

interdisciplinarité ; sciences sociales ; sciences techniques; connaissance ; enquête
Résumé - Cet article est le fruit d'une analyse réflexive d'un collectif pluridisciplinaire de chercheurs qui travaillent par enquête auprès des professionnels de l'agriculture et de la nature. L'objectif est d'apporter des éléments de réflexion sur le rôle des connaissances d'acteurs dans la construction des connaissances scientifiques. La façon de les utiliser varie selon les disciplines, mais plus encore suivant la finalité des recherches. Trois idéaux types sont distingués : décrire et analyser les connaissances des acteurs (1) pour comprendre une situation, (2) pour produire de nouvelles connaissances sur des processus biotechniques, (3) pour accompagner des processus d'action. Cette exploration méthodologique montre combien le recours à l'enquête modifie profondément le contenu cognitif des connaissances agronomiques, éthologiques, écologiques et zootechniques produites et permet aux chercheurs d'innover dans leurs champs disciplinaires respectifs. L'analyse transversale conduit à une réflexion sur le potentiel heuristique de l'enquête.

\section{Keywords:} interdisciplinarity; social sciences; biotechnical sciences; knowledge; surveys

\begin{abstract}
Using professional knowledge in building scientific knowledge; a comparative analysis of several case studies. This paper results from cross-disciplinary reflection by social and biotechnical researchers engaged in surveys with experts from the agriculture and nature spheres: farmers: technical advisors, managers of nature conservation programmes and biologists. The purpose was to analyse the way professional knowledge is used by researchers to enrich their body of scientific knowledge. How professional knowledge is used depends in part on each scientific discipline, but far more on the final research aims. We first identified three ideotypes based on whether professional knowledge is used (1) in a comprehensive way to understand its origins, (2) to build new biological knowledge complementary to the one produced on an experimental basis, or (3) to build operational knowledge to help professionals manage their problems. A cross-analysis of the survey methods used then led to discussing the nature of the scientific knowledge developed from professional knowledge and the innovative potential of such surveys. Finally the heuristic potential of surveys is highlighted.
\end{abstract}

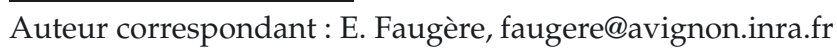




\section{Introduction}

Cet article est le fruit du travail d'un collectif pluridisciplinaire de chercheurs qui s'interrogent sur la construction de leurs connaissances scientifiques ${ }^{1}$. Au-delà de la diversité de nos disciplines respectives (agronomie, zootechnie, éthologie, écologie et ethnologie), nous avons en commun d'étudier des objets frontières ou hybrides mêlant processus sociaux et biologiques, et de mener des enquêtes auprès d'acteurs sociaux professionnels de l'agriculture et/ou de la biodiversité ${ }^{2}$.

L'article analyse le rôle que jouent les connaissances ${ }^{3}$ d'acteurs dans nos démarches scientifiques. Il interroge les différences et les complémentarités entre connaissances d'acteurs et de scientifiques (Darré et al., 2004). En quoi les connaissances et les objets de recherche des disciplines scientifiques sont-ils enrichis par les connaissances d'acteurs, issues de l'observation en situation d'action et conceptualisées (Kolb, 1984), notamment au sein de réseaux sociaux? S'il existe une abondante littérature décrivant les dispositifs sociaux qui mettent en synergie connaissances scientifiques et non scientifiques, ainsi que les modalités d'apprentissage des acteurs (LEARN Group, 2000), peu d'articles traitent des façons concrètes de produire des connaissances scientifiques et de monter en généralité à partir de connaissances d'acteurs. Quels acteurs et quelles connaissances sont mobilisés? Quelle est la nature des connaissances produites? Sontelles des connaissances hybrides, mélanges hétérogènes de connaissances scientifiques et non scientifiques ? Comment sont-elles validées?

Nous avons donc comparé la façon dont nous sommes interrogés, dans nos recherches, par les connaissances des acteurs, en distinguant trois objectifs aux enquêtes que nous menons : décrire et analyser les connaissances des acteurs (1) pour comprendre une situation, (2) pour produire de nouvelles connaissances sur des processus biotechniques ou encore (3) pour accompagner des processus d'action. Nous décrirons pour chacun comment s'effectue le choix des enquêtés, quelle est la nature des connaissances mobilisées, comment s'opèrent la montée en généralité et la production de connaissances scientifiques, et quel est le positionnement disciplinaire de ces dernières.

\footnotetext{
${ }^{1}$ Cet article s'appuie sur un travail collectif, réalisé en 2006 et 2007, au sein d'un atelier méthodologique organisé par Elsa Faugère et Mireille Navarrete dans notre unité de recherche. Le récit concret de nos méthodes d'enquête nous a permis d'avoir des échanges constructifs valorisant l'hétérogénéité de nos disciplines et de nos thématiques de recherche.

2 Agriculteurs, bergers, forestiers, techniciens agricoles, gestionnaires d'aires protégées, biologistes.

3 Nous utiliserons dans cet article le terme «connaissances » plutôt que «savoir», pour insister sur la diversité de nature de ces informations suivant les acteurs qui les produisent ou les utilisent.
}

Nous insistons sur le fait que chacune de ces postures n'est pas l'apanage d'une seule discipline et qu'un chercheur peut, à un moment ou un autre du déroulement de sa recherche, mobiliser plusieurs de ces démarches. Néanmoins, pour la clarté de l'exposé, nous avons regroupé les cas d'étude où un des trois objectifs est largement prépondérant.

\section{Trois démarches de mobilisation des connaissances des acteurs}

\section{Objectif 1 : décrire et analyser les connaissances des acteurs dans une perspective compréhensive}

\section{L'objectif des recherches}

Les ethnologues de notre collectif étudient des mondes sociaux qui sont dans un rapport professionnel avec la nature. Dans un cas, il s'agit de comprendre les transformations du rapport au vivant chez des arboriculteurs; dans l'autre, les évolutions des métiers de gestionnaire d'aires protégées et de taxonomiste (Faugère, 2010, 2008a et b). Dans les deux cas, elles s'appuient sur les connaissances des acteurs dans une perspective compréhensive.

Contrairement aux recherches correspondant aux objectifs 2 et 3 , celles-ci ne concernent pas seulement ni prioritairement les connaissances professionnelles, au sens strict du terme, des acteurs enquêtés (comme les connaissances que les arboriculteurs possèdent sur les techniques de protection des vergers, ou les connaissances taxonomiques des botanistes). Le cœur des investigations concerne les connaissances que ces professionnels possèdent sur le contexte social, politique, économique ou culturel au sein duquel ils exercent leur métier et construisent leurs connaissances professionnelles. Il s'agit donc de décrire, comprendre et analyser ces connaissances dans leur contexte et dans leur historicité, plutôt que d'en saisir le contenu cognitif.

Les ethnologues sont ici dans un travail d'interprétation de leur matériel empirique, constitué, entre autres, des savoirs des professionnels étudiés. Ce travail interprétatif empiriquement ancré est "un surplus, un rajout par rapport à ce qui se dit ou s'interprète déjà ordinairement dans le monde social» (Lahire, 1996). Mais, contrairement à ce que dit la sociologie critique (Bénatouil, 1999), ce surplus interprétatif n'opère pas un travail de dévoilement des rapports de force et de domination dans lesquels seraient pris, à leur insu, les acteurs sociaux étudiés. Il s'inscrit plutôt dans un héritage ancien de l'anthropologie qui, depuis Malinowski (1989), prétend, en partant des catégories et des discours des acteurs, proposer une interprétation des mondes sociaux ou des processus étudiés. Ce qui distingue cette interprétation des autres, et notamment de celles des acteurs eux-mêmes, c'est son 
caractère empiriquement contraint, produit par un dispositif d'enquêtes qualitatives ( $c f$. notamment Olivier de Sardan [1996] et Lahire [1996]). On se situe donc bien dans une tradition sociologique et anthropologique ancienne «selon laquelle les sciences sociales peuvent atteindre le «vrai réel » de l'action [...] sur le plan du sens intime que l'acteur donne à ce qu'il fait [...]» (Dodier, 1991).

\section{Les connaissances d'acteurs mobilisées et leur analyse}

Dans le programme de recherches sur la conservation des forêts sèches de Nouvelle-Calédonie, le choix des acteurs enquêtés était lié aux questions de départ que se posait l'ethnologue :

- comprendre comment et pourquoi, historiquement, s'est constitué un intérêt scientifique pour les forêts sèches ;

- comprendre le fonctionnement actuel du programme, ses enjeux et son organisation.

Pour apporter des éléments de réponse, les personnes les plus compétentes semblaient être, d'une part, les naturalistes qui avaient joué un rôle-clé dans la connaissance scientifique des forêts sèches et, d'autre part, le directeur du programme de conservation de ces forêts. Les premiers entretiens étaient centrés sur la connaissance qu'ils avaient de l'histoire de ce programme, ce qui a notamment conduit à explorer l'histoire de la construction du savoir naturaliste sur la Nouvelle-Calédonie. Mais des acteurs non scientifiques ont également été enquêtés : des élus, des agents des collectivités locales impliquées dans le programme, des propriétaires de forêts sèches, des membres d'associations environnementalistes et d'ONG de la conservation, etc. L'objectif de l'enquête était de croiser les récits, les discours de ces différents acteurs, afin d'obtenir une pluralité de versions sur une même histoire, sur un même processus social, ce que Olivier de Sardan (2003) appelle la «triangulation des données ».

$\mathrm{Au}$ début d'une nouvelle enquête, l'ethnologue est toujours en situation d'apprenant. Il cherche à "rendre compte du point de vue indigène ». Et, pour ce faire, il doit commencer par traduire les questions qu'il se pose au départ, qui généralement ne font pas sens pour les personnes enquêtées, en questions émiques, c'est-à-dire en questions qui ont du sens pour elles (Olivier de Sardan, 1998). Comme le dit Schwartz (1993), «le premier objectif de l'enquête n'est pas de répondre à des questions mais de découvrir celles que l'on va poser, et il faut, pour cette découverte, du temps ». Ce travail de traduction repose précisément sur le recueil des discours des enquêtés, qui vont progressivement lui faire prendre conscience de ce qui fait sens pour eux.

Au cours des premiers entretiens, l'ethnologue a peu d'esprit critique et peu de moyens pour interpréter les propos de l'interlocuteur. Mais, au fil des entretiens, il découvre la diversité des points de vue, des représentations, des controverses, des enjeux, des argumentations sur le sujet. Il identifie progressivement les discours et représentations émiques sur le sujet étudié. Et il se fait alors une idée de plus en plus précise de cette diversité. Ce qui lui permet alors de poser des questions plus pertinentes, de se construire son propre point de vue et de l'argumenter davantage.

\section{Conséquences sur les connaissances scientifiques et les disciplines}

La production des connaissances ethnologiques est intimement liée à la phase de recueil des connaissances des acteurs et à leur interprétation. L'ethnologue va monter en généralité à partir des interprétations sur l'émique qu'il effectue. Ces interprétations vont alimenter un ou des questionnements ethnologiques théoriques. Dans cette étude, qui montre comment la découverte, en 1988, d'une nouvelle espèce végétale (Pittosporum tanianum) a joué un rôle-clé, ce questionnement porte sur les usages sociaux et politiques des savoirs scientifiques et naturalistes. S'il importait peu à l'ethnologue de connaître la classification systématique de cette espèce et sa phylogénie, il lui était en revanche primordial d'observer que cette découverte avait joué un rôle déterminant dans la création du programme de conservation des forêts sèches en 2001. Ce sont donc bien les effets sociaux et politiques de ces connaissances qui intéressaient l'ethnologue, et non leur contenu scientifique.

Dans d'autres cas, lorsqu'un ethnologue travaille sur des sujets où les connaissances techniques et scientifiques sont relativement importantes, la question se pose de savoir jusqu'où il doit aller dans l'acquisition de ces connaissances. Par exemple, lorsque l'une des ethnologues de notre collectif se plonge dans le monde des arboriculteurs en participant à des stages de taille des arbres ou à des journées d'information sur les produits phytosanitaires, il ne s'agit pas pour elle de connaître, comme le ferait un arboriculteur ou un agronome, tous les produits phytosanitaires, leurs efficacités respectives ou leurs conditions d'application. Il s'agit de sélectionner ceux qui font sens socialement : par exemple, ceux qui vont être interdits, parce qu'ils posent la question des alternatives possibles et qu'ils permettent de comprendre le rapport des arboriculteurs à la nature. Mais cela suppose de comprendre suffisamment le domaine technique pour identifier ce qui fait sens pour les professionnels.

La question du niveau de connaissances techniques à acquérir pour enquêter se pose de manière générale pour les ethnologues. Mais elle se pose avec plus d'acuité lorsque l'ethnologue collabore avec des chercheurs de sciences biotechniques, qui ont tendance à questionner l'ethnologue sur sa démarche et sa légitimité à travailler 
sur le sujet. Inversement, l'ethnologue peut questionner la nature des connaissances produites par les chercheurs biotechniciens ou la façon dont elles sont produites. Dans ce cas, les connaissances techniques sont donc à la fois le lien entre les disciplines humaines et biologiques et un lieu éventuel de controverses.

\section{Objectif 2 : décrire et analyser les connaissances des acteurs pour produire de nouvelles connaissances sur les processus biotechniques}

\section{L'objectif des recherches}

En rupture avec les méthodes classiques de production de connaissances scientifiques dans le domaine du vivant, mais dans la lignée de travaux engagés au début des années 1990, notamment au sein du département SAD de l'Inra ( $c f$. notamment Landais et Deffontaines [1989] et Landais [1992], les chercheurs engagés dans cette posture cherchent à valoriser les connaissances des acteurs pour produire de nouvelles connaissances sur des processus biotechniques. C'est le cas lorsqu'ils étudient le comportement d'un troupeau en interaction avec le berger (Lécrivain, 2004), les effets des pratiques culturales sur le développement des maladies en maraîchage (Tchamitchian et al., 2008) ou sur la qualité des fruits (Fauriel et al., 2007). Les connaissances des acteurs sont analysées dans le champ scientifique, et donc confrontées à la théorie (éthologique ou agronomique) pour l'enrichir, l'affiner et formuler de nouvelles hypothèses. Contrairement aux ethnologues du groupe, ces chercheurs se centrent sur le contenu cognitif des connaissances des bergers ou des agriculteurs, mais n'étudient pas ou peu leur origine, leur histoire, la façon dont elles évoluent. Ce faisant, ils se placent dans une perspective étique, au sens où l'étique repose sur des observations externes indépendantes des significations portées par les acteurs (Olivier de Sardan, 1998).

Dans la littérature scientifique, les recherches biotechniques à partir de connaissances d'acteurs proviennent souvent de pays en voie de développement ou émergents. Il s'agit alors de combler les lacunes scientifiques en se basant sur l'expertise locale. Par exemple, Barzman et al. (1996) étudient les savoirs d'arboriculteurs, au Vietnam, sur l'utilisation d'un auxiliaire biologique pour améliorer la qualité des fruits. Ils réalisent des expérimentations agronomiques pour expliciter les processus biologiques sous-jacents aux savoirs des arboriculteurs locaux et pour définir leur niveau de généricité. Quelques études ont porté sur des agrosystèmes de pays développés, mais pour des espèces rares où peu d'expertise scientifique existe (Girard et Navarrete, 2005). L'originalité de nos travaux tient à la volonté d'utiliser ces connaissances validées dans une perspective opérationnelle de conception de systèmes de culture.

\section{Les connaissances d'acteurs mobilisées et leur analyse}

Les chercheurs engagés dans cette démarche choisissent volontairement d'enquêter des professionnels expérimentés ou novateurs :

- des agriculteurs qui expérimentent depuis plusieurs années des modes de conduite originaux ;

- des bergers qui pilotent le déroulement des interactions troupeau-territoire et qui ont acquis un sens aigu de l'observation des milieux à pâturer et des animaux.

Les chercheurs accèdent ainsi à des connaissances précises et à des modes de raisonnement proches des leurs. Raeddeke et Rikkon (1997) ont identifié deux types d'agriculteurs : ceux qui évaluent de nouvelles pratiques agricoles sur la base de leur compatibilité avec leurs expériences agricoles et leurs modes de pensée; ceux qui les évaluent sur la base de comparaisons dans une démarche expérimentale. Les agronomes et l'éthologue postulent que les savoirs des seconds peuvent être plus facilement intégrés à leurs savoirs théoriques disciplinaires. Ils mobilisent également les savoirs de conseillers techniques, qui ont des représentations synthétiques (issues de l'analyse d'observations faites chez différents agriculteurs ou éleveurs), première étape dans un processus de formalisation des connaissances. Dans cette démarche, peu importe donc que les acteurs soient représentatifs ou non, reconnus socialement (au sens de Darré [1999]) ou non ; c'est bien la démarche réflexive qu'ils ont sur leurs pratiques et la pertinence de leur raisonnement pour les scientifiques qui priment.

Prenons l'exemple d'une recherche portant sur le contrôle des maladies dans les systèmes maraîchers (Tchamitchian et al., 2008). La finalité de ce travail est de concevoir de nouveaux systèmes techniques, mais aussi d'accroître les savoirs méthodologiques sur l'utilisation des connaissances d'acteurs dans une discipline qui y recourt encore peu.

L'analyse de ces connaissances repose sur le postulat, classique en sciences expérimentales, qu'une même cause produit les mêmes effets. C'est ce postulat qui oriente le recueil et le traitement des connaissances des agriculteurs. L'enquête repose sur un questionnaire relativement précis pour caractériser le système sur les plans biologique et technique (caractérisation des états du sol, des pratiques mises en œuvre, de l'état pathologique des cultures), ainsi que sur un entretien ouvert pour accéder aux liens que font les agriculteurs entre leurs systèmes techniques et les maladies. En comparant un grand nombre de parcelles, l'agronome identifie 
celles qui ont des états biologiques et techniques voisins, puis compare les niveaux de contamination estimés par les agriculteurs. Si une même relation est exprimée par plusieurs agriculteurs, le chercheur la considère comme une nouvelle connaissance scientifique potentielle et vérifie sa compatibilité avec l'état actuel des connaissances scientifiques. Mais il peut aussi identifier des connaissances en apparence contradictoires. Par exemple, certains agriculteurs considèrent que « les nématodes font des dégâts sur toutes les cultures de salade, d'octobre à avril ", et d'autres que «seules les salades récoltées en novembre-décembre sont attaquées ». Mobilisant ses connaissances théoriques et les données disponibles sur les deux cas, l'agronome trouve une cohérence aux deux discours : pour un faible inoculum du sol, les dégâts ne sont détectés qu'à la période de multiplication des nématodes la plus forte, c'est-à-dire en automne. Mais lorsque le niveau de contamination du sol dépasse un certain seuil, des dégâts sont observés même en plein hiver, bien que le froid limite la multiplication de ces ravageurs. L'observation d'un plus grand nombre de situations et la mesure des dégâts sur culture et du niveau d'infestation dans le sol peuvent alors permettre de valider cette hypothèse et de préciser ce seuil d'infestation.

La prise en compte de connaissances d'acteurs peut conduire à élargir les contours d'une discipline biotechnique. C'est le cas pour l'éthologue de notre groupe. Pendant longtemps, le principe de base de l'éthologie a été de faire de l'observation dite objective, l'idéal étant d'être derrière une glace sans tain. Pour l'observation du comportement d'animaux au pâturage, la présence d'un berger au milieu du troupeau était vécue comme un artefact inacceptable. Mais pour étudier l'importance des conditions d'élevage dans l'expression des comportements des troupeaux, l'éthologue a été amenée à prendre en compte les savoir-faire des bergers quand ils gardent leurs troupeaux (Lécrivain, 2004). Une enquête a été réalisée dans l'arrière-pays méditerranéen auprès d'une vingtaine de bergers ayant plus de dix années de garde dans des milieux variés. Elle a reposé, d'une part, sur l'observation de l'activité des bergers en situation de garde, d'autre part, sur des entretiens semi-directifs portant sur les raisons du choix des pratiques, la manière de concevoir le rôle du berger et celui du chien, les indicateurs du comportement des brebis et du troupeau utilisés, les façons d'agir sur le troupeau et leur prise en compte du territoire. En accédant ainsi aux conceptions des bergers, l'éthologue a mis en lumière des indicateurs de gestion du comportement animal utilisés pour la garde des troupeaux, ce qu'elle n'aurait pas pu faire auparavant.

Elle a donc opéré, progressivement, un glissement disciplinaire et un élargissement des contours de sa discipline d'origine, l'éthologie : d'une part, en s'intéressant aux interactions des ruminants domestiques au pâturage avec les humains ; d'autre part, en éclairant sa compréhension du comportement animal par les savoirs des bergers.

\section{Conséquences sur les connaissances scientifiques et les disciplines}

Dans les deux derniers exemples, de nouvelles connaissances agronomiques et éthologiques ont été élaborées par les scientifiques en s'appuyant sur des connaissances exprimées par les professionnels : identification d'interactions entre système de culture et niveau d'infestation des nématodes en maraîchage; prise en compte des capacités de repérage spatial des brebis et du comportement du troupeau pour en organiser la garde. À partir de connaissances établies dans une situation particulière (une exploitation, un troupeau, un milieu), le scientifique produit des connaissances plus génériques, ou du moins avec un domaine de validité identifié. Dans le cas du maraîchage, ces connaissances servent à coconstruire de nouveaux systèmes techniques avec les agriculteurs. Dans le cas du pâturage, elles sont transmises à des bergers en situation d'apprentissage, ou à des gestionnaires d'écosystèmes pour proposer de nouveaux modes de gestion des espaces naturels. Cette intégration de connaissances des acteurs dans une démarche biotechnique pour concevoir des innovations techniques est peu décrite dans la bibliographie et reste novatrice. Il existe cependant des travaux pionniers, comme ceux, en France, de chercheurs du département SAD de 1'Inra - Landais et Deffontaines (1989) - , qui furent suivis par des recherches innovantes sur les pratiques d'élevage extensives - Landais (1992). Ce type de démarche contribue à une réflexion méthodologique :

- en agronomie, la synergie entre des observations systémiques des professionnels et des connaissances plus analytiques des scientifiques élargit la gamme des méthodes utilisables dans la discipline. Par exemple, Girard et Navarrete (2005) proposent une lecture critique de l'analogie entre espèces, qui est fréquemment faite par les professionnels, afin de la rendre plus pertinente scientifiquement ;

- en éthologie, l'intégration des pratiques de gardiennage aux démarches classiques d'observation du comportement des troupeaux conduit à considérer l'homme comme un élément du système, posture favorisant la compréhension des réactions des uns par rapport aux autres. Le dispositif d'observation et d'enquête permet de s'intéresser à la façon dont se construisent les interactions troupeau-territoireberger, d'analyser la coproduction de comportements et d'explorer l'interprétation par les bergers de ce que font les troupeaux - telle est la posture de l'éthologue Thelma Rowell décrite par Vinciane Despret (2002). 


\section{Objectif 3 : formaliser et rendre visibles les connaissances des acteurs pour produire des connaissances opérationnelles}

\section{L'objectif des recherches}

Les chercheurs impliqués dans cette démarche d'accompagnement étudient les transformations des activités agricoles, puis ils construisent des méthodes et des outils pour renforcer les acteurs dans la maîtrise de ces transformations. Ils produisent donc des connaissances actionnables, c'est-à-dire de nature à renforcer les acteurs dans la conduite de leurs activités (Avenier et Schmitt, 2007).

Un premier exemple porte sur l'accompagnement des changements de pratiques d'éleveurs caprins en vue de développer l'utilisation du pâturage. Il s'agit là d'un enjeu pour les éleveurs qui veulent limiter les charges, améliorer l'image du produit et mieux valoriser le territoire. Mais le retour au pâturage implique un changement de logique et la gestion de nouveaux risques et incertitudes (climatiques, alimentaires, sanitaires...). Pour le chercheur, il ne s'agit donc pas de proposer le bon modèle à appliquer, robuste quel que soit le contexte, mais de construire un cadre d'analyse qui aide l'éleveur à comprendre sa propre situation, à raisonner sur les évolutions de la conduite du troupeau pour ajuster ses pratiques chemin faisant.

\section{Les connaissances d'acteurs mobilisées et leur analyse}

Dans cette recherche d'ordre méthodologique, le choix des enquêtés s'est porté sur des éleveurs pour lesquels la gestion du pâturage était un problème et qui étaient prêts à s'impliquer dans la recherche de solutions. Les chercheurs ont réalisé des entretiens ouverts auprès des éleveurs, basés sur la narration de leurs modes de conduite et de leurs évolutions. Ces entretiens sont structurés en deux temps (Napoléone, 2004). Dans un premier temps, l'éleveur décrit sur un mode chronologique sa conduite du troupeau tout au long de l'année, qui est formalisée sur un support calendaire graphique. Au cours de cette narration, sont enregistrés également les divers événements survenus au niveau du troupeau et du contexte agricole ainsi que la façon dont l'éleveur a apprécié, sur le moment, la situation. Le chercheur identifie alors des connaissances techniques particulières mobilisées par l'éleveur pour conduire son exploitation (par exemple, intérêt de tel type de ressource à tel moment compte tenu de la production du troupeau). Dans un second temps, l'objectif est de prendre du recul sur la narration, de formaliser la stratégie de production de l'éleveur et d'évaluer sa mise en œuvre. Le support graphique devient alors une représentation matérielle facilitant l'échange entre le chercheur et l'éleveur, et joue le rôle de médiation. Ici, ce qui est au cœur de l'investigation, c'est donc le lien entre les systèmes techniques et la perception que les éleveurs ont de ces systèmes ; il s'agit de caractériser la diversité des pratiques de l'éleveur, des modalités de mise en œuvre, des règles et des indicateurs de gestion utilisés, des moments critiques et des manières de réagir.

Un deuxième exemple porte sur la gestion d'une ressource commune par un collectif d'acteurs. Il permet d'illustrer comment l'écologue de notre collectif s'est engagé dans une nouvelle démarche, appelée «modélisation d'accompagnement » (Collectif ComMod, 2006 et 2009). Il est progressivement passé d'une écologie «pure et dure », qui ne voyait dans les activités humaines qu'un facteur de perturbation des dynamiques naturelles de la végétation qu'il étudiait, à une approche qui tente de modéliser de façon participative les interactions entre les dynamiques écologiques et les pratiques des hommes. Les systèmes multi-agents lui ont permis de mettre en relation des modèles biophysiques avec des modèles de décision, et ainsi de réaliser cette articulation.

Au cours de cette exploration, il a pratiqué l'interdisciplinarité en confrontant les points de vue de biologistes, d'écologues, de généticiens, de géographes, de sociologues ou d'ethnologues. Dans cette démarche, les connaissances des acteurs sur les processus en jeu sont autant prises en compte que les connaissances scientifiques; la coconstruction de modèles avec des chercheurs, des techniciens et des acteurs locaux aboutit à des connaissances hybrides, qui regroupent dans un formalisme commun tout ce qui a été jugé pertinent par le collectif. Dans ce processus de coconstruction, le choix des acteurs invités, leur représentativité et l'équité dans l'accès à la parole sont des questions centrales qui doivent systématiquement être posées à l'initialisation de la démarche (Étienne, 2009). Ainsi, en prenant en compte les dynamiques humaines, en cherchant à en modéliser les règles de décision et les modalités d'adaptation, il est volontairement sorti de ses frontières disciplinaires; il a pris des risques, mais il s'est aussi démarqué des «courants classiques » de l'écologie, en ouvrant un nouveau front de recherche dans la biologie de la conservation (Garnier, 2008 ; Hervé et Laloë, 2009).

\section{Conséquences sur les connaissances scientifiques et les disciplines}

Ces travaux débouchent sur plusieurs productions scientifiques :

- des connaissances scientifiques, par exemple la caractérisation des systèmes techniques, de leurs transformations, de leur diversité, des logiques sous-jacentes ; - des productions méthodologiques : méthodes d'entretien et d'aide à l'expression de projet, à la confrontation de points de vue; démarches et modèles d'accompagnement des changements de pratiques ; 
- des interrogations sur les modèles des scientifiques. Par exemple, en zootechnie, le modèle de rationnement qui tient compte des besoins des animaux et des apports nutritifs des aliments distribués n'est pas utilisable dans l'alimentation au pâturage, où l'éleveur ne gère pas la qualité nutritionnelle de chaque élément consommé. D'où un autre modèle, qui ne se réfère non pas à un corpus de connaissances académiques, mais à leur pertinence au regard de l'action elle-même (Avenier, 1992) ;

- une modification des contours de la discipline, qui participe à la création d'une nouvelle discipline, comme la modélisation d'accompagnement.

\section{Discussion : conséquences épistémologiques et méthodologiques}

Nous aborderons successivement deux points : une réflexion sur nos perspectives émiques, étiques et/ou opérationnelles ; une réflexion épistémologique sur ce qu'entraîne le recours aux connaissances des acteurs dans nos démarches scientifiques ${ }^{4}$.

\section{L'émique, l'étique, l'opérationnel}

Bien que, par souci de clarté, nous ayons présenté successivement les trois démarches à partir d'exemples distincts, notre propos est ici d'illustrer les interrelations qui existent entre les trois postures.

Dans la perspective émique, il s'agit de recueillir et d'analyser les savoirs des enquêtés pour comprendre des processus sociaux et restituer la vision du monde qu'en ont les acteurs (objectif 1). Le chercheur monte en généralité par un travail d'interprétation de son matériel empirique qui consiste à confronter ce matériel aux théories existantes. Mais cette perspective émique ne se limite pas aux seuls ethnologues de notre collectif, puisque des chercheurs relevant des disciplines biotechniques mobilisent parfois aussi cette démarche, même si elle n'est pas l'objectif principal de leur recherche. C'est le cas de l'éthologue (objectif 2) et des zootechniciens en posture d'accompagnement (objectif 3), qui décryptent le sens

\footnotetext{
${ }^{4}$ La perspective étique désigne une analyse extérieure du fonctionnement des sociétés et des cultures, par opposition à une analyse "du dedans », qui tente d'élucider comment les hommes classent, ordonnent, perçoivent et, à travers ces grilles d'analyse, utilisent le monde qui les entoure (Bromberger, 1986). «K.-L. Pike oppose ces deux démarches, quand il distingue le point de vue «étique »-celui de l'observateur qui plaque une grille d'analyse préconstituée sur la réalité - et le point de vue «émique»-qui retient uniquement les traits pertinents pour l'usager (l'opposition «étique »/《émique » renvoie aux différences fondamentales de méthodes entre la phonétique et la phonologie, phonemics » (ibid.).
}

que les acteurs donnent à leurs pratiques, leurs modes de raisonnement et leurs manières de faire pour produire des connaissances opérationnelles.

Les chercheurs impliqués dans un objectif de production de connaissances biotechniques se placent dans une perspective étique, mais ils le font de façon un peu différente suivant la posture qu'ils retiennent pour conduire leurs activités. Dans le cas de la lutte contre les pathogènes en maraîchage, le chercheur dissocie partiellement la production de connaissances biotechniques (relation entre système de culture et maladies, décrite dans l'objectif 2) et la production de connaissances opérationnelles (coconception avec les acteurs de systèmes techniques, qui renvoie à l'objectif 3). Dans la première étape, il se focalise sur l'analyse des connaissances que les agriculteurs ont sur leurs cultures ; cela révèle des phénomènes, des indicateurs qu'il n'a pas encore étudiés. Il peut parfois se trouver confronté à des connaissances d'acteurs trop en rupture avec le socle des connaissances scientifiques pour pouvoir l'alimenter. Ces connaissances ne sont alors, momentanément du moins, d'aucune utilité pour le chercheur. Dans le cas de l'utilisation du pâturage en production caprine (décrit dans l'objectif 3), les connaissances des acteurs conduisent le chercheur à revisiter ses propres connaissances zootechniques, pour proposer de nouveaux indicateurs, de nouvelles façons de caractériser les processus biotechniques et leur diversité (d'où un retour à l'objectif 2).

Dans la perspective opérationnelle, il s'agit de comprendre et de partager les façons de voir des acteurs, pour construire avec eux une lecture de la situation actuelle et chercher ensemble des solutions. Ce faisant, on construit des outils dans une perspective émique, comme la construction de représentations partagées, qui permettent de faire émerger les points de vue des acteurs et la signification qu'ils donnent aux pratiques (Hatchuel, 1994; Vinck, 1999). Mais il s'agit aussi de formaliser des référentiels collectifs, qui peuvent ensuite être mobilisés dans les processus d'action. Par exemple, si l'on met en place telle pratique dans telle zone, alors la ressource va évoluer de telle manière. Ces savoirs sont formulés dans une acception qui a du sens pour les acteurs au regard de leurs préoccupations et des processus qu'ils gèrent.

\section{Quelles connaissances scientifiques produites sur la base des connaissances d'acteurs?}

Quelles que soient leurs disciplines et leurs finalités, au cours de leurs enquêtes, les chercheurs de notre collectif se placent délibérément dans une situation d'apprenant. Ils puisent auprès des acteurs leurs connaissances des processus biophysiques ou sociaux, et de leurs interactions, pour progressivement valider, modifier et/ou élaborer des connaissances scientifiques. Ainsi, dans le 
débat d'idées actuel sur les interactions entre acteurs sociaux et scientifiques dans la production scientifique, on se situe bien ici dans le modèle de la coproduction des savoirs (Callon, 1998), suivant quatre orientations :

- la production de nouvelles connaissances théoriques dans la discipline d'origine du chercheur. C'est notamment le cas des ethnologues (sur les rapports nature-culture), des agronomes (sur les conditions d'élaboration de la production végétale) et de l'éthologue (sur la gestion par les bergers du comportement spontané des brebis);

- la production de connaissances méthodologiques dans la discipline d'origine du chercheur. C'est notamment le cas des agronomes de notre collectif, qui s'interrogent sur les formes de validation de ces nouvelles connaissances agronomiques issues des enquêtes ;

- une modification des contours de la discipline d'origine du chercheur. C'est le cas de l'écologue et de l'éthologue, qui cherchent tous deux à intégrer les humains dans leurs analyses des écosystèmes. C'est aussi le cas dans la démarche d'accompagnement des processus d'action, où les connaissances méthodologiques produites mobilisent divers corpus disciplinaires. Est-ce là une nouvelle «science de la conduite des cultures et de l'élevage », aux marges de plusieurs disciplines, comme le suggère Jollivet (2004)?

- une production dans le champ d'une autre discipline. C'est le cas de l'écologue, qui produit des méthodes dans le domaine de l'accompagnement, lesquelles relèvent des sciences de l'accompagnement et/ou de la complexité.

Cette exploration méthodologique montre combien le recours à l'enquête a profondément modifié le contenu cognitif des savoirs agronomiques, éthologiques, écologiques et zootechniques produits grâce aux acteurs et permis aux chercheurs d'innover dans leurs champs disciplinaires respectifs. Il y a donc là un fort potentiel de découverte, qui varie notamment en fonction des acteurs enquêtés. Certains d'entre eux, placés dans des situations particulières, sont poussés à adopter des pratiques innovantes. Le chercheur utilise alors l'inventivité des enquêtés pour explorer de nouvelles hypothèses scientifiques. Cela pose d'ailleurs la question de la reconnaissance du rôle de ces acteurs dans la production de connaissances scientifiques et des bénéfices qu'ils peuvent, en retour, y trouver.

Enfin, en sortant du cœur de leur discipline d'origine pour en explorer les frontières, les chercheurs prennent le risque d'être marginalisés au sein de leur discipline et de ne pas être reconnus, malgré l'exploration de nouvelles frontières disciplinaires.

Pour conclure, cet article et l'atelier dont il est issu montrent tout l'intérêt que peuvent avoir des discussions méthodologiques pour des chercheurs engagés dans des démarches interdisciplinaires. En effet, cette comparaison fine des méthodes de travail a permis un échange constructif entre des chercheurs issus de disciplines très différentes, alors que ceux-ci étaient engagés sur des thématiques et des objets également divers. Ainsi, le partage d'expérience sur les positionnements étique, émique et opérationnel a permis à chacun de clarifier les objectifs de chaque phase de recherche et la façon dont, souvent, nous combinons plusieurs d'entre eux, simultanément ou séquentiellement. Mais, plus globalement, cette comparaison contribue aussi à «faire progresser méthodologiquement la façon d'assembler, voire d'intégrer les apports disciplinaires », enjeu essentiel de toute recherche interdisciplinaire, comme le soulignent Jollivet et Legay (2005).

\section{Références}

Avenier, M.-J., 1992. Recherche-action et épistémologies constructivistes, modélisation systémique et organisations socio-économiques complexes, Revue internationale de systémique, 6, 4, 403-420.

Avenier, M.-J., Schmitt, C. (Eds), 2007. La Construction de savoirs pour l'action, Paris, L'Harmattan.

Barzman, M.S., Mills, N.J., Nguyen Thi Thu Cuc, 1996. Traditional knowledge and rationale for weaver ant husbandry in the Mekong delta of Vietnam, Agriculture and Human Values, 13, 4, 2-9.

Bénatouil, T., 1999. Critique et pragmatique en sociologie. Quelques principes de lecture, Annales. Histoire, sciences sociales, 54, 2, 281-317.

Bromberger, C., 1986. Les savoirs des autres, Les Hommes et le milieu naturel, Terrain, 6, mis en ligne le 19 juillet 2007, URL : http://terrain.revues.org/index2890.html (consulté le 4 novembre 2010).

Callon, M., 1998. Des différentes formes de démocratie technique, Les Annales des mines / Responsabilité E Environnement, 9, 63-73.

Collectif ComMod, 2006. Modélisation d'accompagnement, in Amblard, F., Phan, D. (Eds), Modélisation et simulation multiagents : applications aux sciences de l'homme et de la société, Londres, Hermes Science Publications, 217-228.

Collectif ComMod, 2009. La posture d'accompagnement des processus de prise de décision : les références et les questions transdisciplinaires, in Hervé, D., Laloë, F. (Eds), Modélisation de l'environnement : entre natures et sociétés, Versailles, Quæ, 71-89.

Darré, J.-P., 1999. La Production de connaissances pour l'action : arguments contre le racisme de l'intelligence, Paris, Éditions de la MSH / INRA Éditions.

Darré, J.-P., Mathieu, A., Lasseur, J. (Eds), 2004. Le Sens des pratiques : conceptions d'agriculteurs et modèles d'agronomes, Paris, INRA Éditions.

Despret, V. 2002. Quand le loup habitera avec l'agneau, Paris, Les Empêcheurs de penser en rond.

Dodier, N., 1991. Agir dans plusieurs mondes, Critique, 529-530, 427-458.

Étienne, M., 2009. Co-construction d'un modèle d'accompagnement selon la méthode ARDI : guide méthodologique, Laudun, Cardère éditeur. 
Faugère, E., 2008a. Mettre en mots, en nombres et en politique la nature néo-calédonienne, Journal de la Société des océanistes, 126-127, 153-166.

Faugère, E., 2008b. L'exploration contemporaine de la biodiversité. Approche anthropologique de l'expédition Santo 2006, Journal de la Société des océanistes, 126-127, 195-205.

Faugère, E., 2010. La fabrication sociale et politique des forêts sèches de Nouvelle-Calédonie (1981-2001), in Faugère, E., Merle, I. (Eds), La Nouvelle-Calédonie vers un destin commun?, Paris, Karthala.

Fauriel, J., Bellon, S., Plenet, D., Amiot, M.-J., 2007. On-farm influence of production patterns on total polyphenol content in peach, Improving Sustainability in Organic and Low Input Food Production Systems, 3rd Int. Congress of QLIF Project, University of Hohenheim, Germany, March 20-23.

Garnier, L. (Ed.), 2008. Entre l'homme et la nature, une démarche pour des relations durables, Paris, Unesco-MAB.

Girard, N., Navarrete, M., 2005. Quelles synergies entre connaissances scientifiques et connaissances empiriques? L'exemple des cultures du safran et de la truffe, Natures Sciences Sociétés, 13, 1, 33-44.

Hatchuel, A., 1994. Apprentissage collectifs et activités de conception, Revue française de gestion, 99, 109-120.

Hervé, D., Laloë, F. (Eds), 2009, Modélisation de l'environnement : entre natures et sociétés, Versailles, Quæ.

Jollivet, M., 2004. Préface, in Darré, J.-P., Mathieu, A, Lasseur, J. (Eds), Le Sens des pratiques : conceptions d'agriculteurs et modèles d'agronomes, Paris, INRA Éditions, 3-7.

Jollivet, M., Legay, J.-M., 2005. Dossier interdisciplinarité. Canevas pour une réflexion sur une interdisciplinarité entre sciences de la nature et sciences sociales, Natures Sciences Sociétés, 13, 2, 184-188.

Kolb, D.A. 1984. Experiential Learning: Experience as a Source of Learning and Development, Englewood Cliffs (NJ), PrenticeHall.

Lahire, B., 1996. Risquer l'interprétation. Pertinences interprétatives et surinterprétations en sciences sociales, Interpréter, surinterpréter, Enquête. Anthropologie, histoire, sociologie, 3, 61-87, mis en ligne le 31 octobre 2007, URL : http://enquete.revues.org/document373.html.

Landais, É. (Ed.), 1992. Pratiques d'élevage extensif : identifier, modéliser, évaluer, Études et Recherches, 27.
Landais, É., Deffontaines, J.-P., 1989. Analysing the management of a pastoral territory. The study of the practices of a shepherd in the southern French Alps. Études $\mathcal{E}$ Recherches, 16, 199-207.

LEARN Group [Cerf, M., Gibbon, D., Hubert, B., Ison, R., Jiggins, J., Paine, M., Proost, J., Roling, N.], 2000. Cow Up a Tree : Knowing and Learning for Change in Agriculture. Case Studies from Industrialised Countries, Paris, INRA Éditions.

Lécrivain, É., 2004. Shepherding : Which know-how and relevance to the present day, XI World Congress of Rural Sociology, International Rural Sociology Association (IRSA), Trondheim, Norway, July 25-30.

Malinowski, B., 1989. Les Argonautes du Pacifique occidental, Paris, Gallimard.

Napoléone, M., 2004. La coproduction d'un diagnostic technique avec des éleveurs caprins, in Darré, J.-P., Mathieu, A., Lasseur, J. (Eds), Le Sens des pratiques : conceptions d'agriculteurs, modèles d'agronomes, Paris, INRA Éditions, 255-273.

Olivier de Sardan, J.-P., 1996. La violence faite aux données : de quelques figures de la surinterprétation en anthropologie, Interpréter, surinterpréter, Enquête. Anthropologie, histoire, sociologie, 3, 31-59, mis en ligne le 31 octobre 2007, URL : http://enquete.revues.org/document363.html.

Olivier de Sardan, J.-P., 1998. Émique, L'Homme, 147, 151-166.

Olivier de Sardan, J.-P., 2003. Observation et description en socio-anthropologie, in Blundo, G., Olivier de Sardan, J.-P. (Eds), Pratiques de la description, Paris, Éditions de l'EHESS, 3, 13-40.

Raeddeke, A.H., Rikoon, J.S., 1997. Temporal and spatial dimensions of knowledge: Implications for sustainable agriculture, Agriculture and Human Values, 14, 145-158.

Schwartz, O., 1993. L'empirisme irréductible, in Anderson, N. (Ed.), Le Hobo, Paris, Nathan, 265-308.

Tchamitchian, M., Navarrete, M., Bressoud, F., Fauriel, J., Pares, L., 2008. Building sustainable cropping systems by combining scientific and empirical knowledge. The example of soil-born disease control on vegetable sheltered crops, First Symposium on Horticulture in Europe, Vienna, Austria, February 17-20.

Vinck, D., 1999. Ingénieur au quotidien : ethnologie de l'activité de conception et d'innovation, Grenoble, PUG.

Reçu le 20 mai 2008. Accepté le 7 octobre 2010. 